\title{
Production des Déchets et Santé des Travailleurs: Cas de la Mine d'or de Tongon (Côte d'Ivoire)
}

\author{
Antoine Sehi Bi Tape, \\ Département de Géographie, \\ Université Peleforo Gon Coulibaly, Korhogo, Côte d'Ivoire \\ Aboubakar Coulibaly, \\ Paul Kouassi Anoh, \\ Jérôme N'guessan Aloko, \\ Institut de Géographie Tropicale, \\ Université Félix Houphouët-Boigny, Abidjan, Côte d'Ivoire
}

Doi:10.19044/esj.2019.v15n32p104 URL:http://dx.doi.org/10.19044/esj.2019.v15n32p104

\section{Résumé}

Cette étude porte sur la production des déchets dans l'exploitation aurifère de Tongon en Côte d'Ivoire. L'objectif visé est d'évaluer l'incidence des déchets produits dans la mine d'or de Tongon sur la santé des travailleurs. La méthodologie de collectes de données était basée sur la recherche documentaire et la réalisation d'une enquête faite à partir d'un questionnaire. Sur la base d'un sondage stratifié, 297 travailleurs ont été retenus pour cette étude. Elle a permis de mettre en évidence les différents types de déchets miniers (ou industriels) produits et les pathologies qui seraient susceptibles d'y être liées. Ces déchets miniers sont constitués de roches stériles (résidus miniers) (82\%), de terres contaminées $(1 \%)$ et de boues $(17 \%)$ renfermant du cyanure, de l'acide chlorhydrique, de l'arsenic, du plomb, du fer, du nitrate, du peroxyde d'hydrogène qui sont très néfastes pour la santé. L'étude révèle aussi que, les pathologies et les signes cliniques tels que les irritations oculaires $(8 \%)$, digestives $(14 \%)$, respiratoires $(11 \%)$ et auditives $(4 \%)$, les céphalées fréquentes (24\%) et les lésions caustiques (17\%) observées chez les travailleurs de cette zone d'étude pourraient être liées à l'exposition de ceuxci et à la manipulation des intrants chimiques.

Mots clés: Activités industrielles, Mine d'or, Santé, Tongon, Côte d'Ivoire 


\title{
Waste Generation and Workers' Health: The Case of the Tongon Gold Mine (Côte d'Ivoire)
}

\author{
Antoine Sehi Bi Tape, \\ Département de Géographie, \\ Université Peleforo Gon Coulibaly, Korhogo, Côte d'Ivoire \\ Aboubakar Coulibaly, \\ Paul Kouassi Anoh, \\ Jérôme N'guessan Aloko, \\ Institut de Géographie Tropicale, \\ Université Félix Houphouët-Boigny, Abidjan, Côte d'Ivoire
}

\begin{abstract}
This study focuses on waste production at the Tongon gold mine in Côte d'Ivoire. The objective is to assess the impact of the waste produced in the Tongon gold mine on the health of workers. The data collection methodology was based on documentary research and a questionnaire-based survey. Based on a stratified survey, 297 workers were selected for this study. It made it possible to identify the different types of mining (or industrial) waste produced and the pathologies that could be linked to it. This mining waste consists of waste rock (mine tailings) (82\%), contaminated soil (1\%) and sludge (17\%) containing cyanide, hydrochloric acid, arsenic, lead, iron, nitrate, hydrogen peroxide which are very harmful to health. The study also reveals that clinical pathologies and signs such as eye (8\%), digestive (14\%), respiratory $(11 \%)$ and auditory (4\%) irritations, frequent headaches $(24 \%)$ and caustic lesions (17\%) observed in workers in this study area could be related to their exposure and to the handling of chemical inputs.
\end{abstract}

Keywords: Industrial activities, Gold mine, Health, Tongon, Ivory Coast

\section{Introduction}

Le sous-sol ouest africain est doté d'importantes ressources minières dont les perspectives de développement sont favorables (Bamba et al., 2008, p.84). Il contient de l'or, du diamant, du pétrole, de l'uranium, du fer, du manganèse, de la bauxite, du phosphate... Sous l'impulsion des politiques minières nationales attractives et d'un fort investissement du secteur privé étranger on assiste depuis quelques années à un développement important du 
secteur minier dans cette partie du continent africain (UICN/PAPACO ${ }^{7}, 2011$, p. 2).

A l'instar des autres pays de la sous-région ouest-africaine, la Côte d'Ivoire, qui est un pays à vocation agricole n'est pas en reste. Elle regorge également d'un potentiel en ressources minières (or, fer, manganèse, bauxite, cobalt, diamant, nickel, cuivre...) ( $\mathrm{CCI}^{8}, 2017$, p. 1). Le code minier qui réglemente ce secteur met un accent particulier sur les impacts environnementaux des projets de développement. Cette législation vise à garantir une bonne gestion de l'environnement, la protection et le bien-être des populations riveraines et des travailleurs et la réalisation des projets dans des conditions idoines au niveau de toutes les activités publiques ou privées $\left(\mathrm{SGS}^{9}, 2016\right.$, p. 26).

Conscient de la baisse des prix des produits agricoles (café-cacao), la Côte d'Ivoire a fait du secteur minier, un secteur stratégique de sa politique de développement et une source importante pourvoyeuse de devises. Ce secteur contribue à hauteur de 2,3\% du Produit Intérieur Brut (PIB) et emploie environ 30000 personnes (CCI, 2017, p. 1). En 2017, 29 nouveaux permis de recherche minière ont été accordés aux entreprises dans tout le pays, ce qui porte à 164, le nombre total de permis de recherche minière. Les ressources financières issues des activités minières se chiffraient à 539 milliards de francs CFA (1,02 milliard de dollars) en 2017 contre 483 milliards (913 millions de dollars,) en 2016. Parmi ces produits miniers, l'or est la substance minérale la mieux présentée avec 138 permis de recherche, soit $84 \%$ du total des permis délivrés. Cet accroissement du nombre des permis miniers a engendré le doublement de la production de l'or qui est passée de 12 tonnes en 2011 à 25,395 tonnes en 2017 (Kone, 2018, pp.1-2).

Nonobstant les retombées économiques issues de l'exploitation des ressources minières (taxes diverses, création d'emplois directs et indirects, création d'entreprises), l'activité minière engendre de nombreux problèmes environnementaux (déforestation, pollution de l'air, des sols et des eaux), socio-économiques (perte et diminution des surfaces cultivables, déplacement et recasement des populations) et sanitaires (émergence et recrudescence de certaines pathologies). Face à ce double enjeu, la gestion durable des déchets constitue dès lors un indicateur de la qualité du cadre de vie et un critère de notation important pour les agences de normalisation.

Située sur le permis d'exploration de Niellé au nord de la Côte d'Ivoire, la mine de Tongon est considérée comme la plus grande exploitation aurifère du pays. Elle a pour principales activités l'exploitation de la carrière et le

${ }^{7}$ Union International pour la Conservation de la Nature/Programme des Aires Protégées d'Afrique Centrale et de l'Ouest

${ }^{8}$ Chambre du Commerce et d'Industrie de Côte d'Ivoire

${ }^{9}$ Société Générale de Surveillance 
traitement des minerais (concassage du minerai, broyage, circuit de lixiviation, circuit d'élution et récupération de l'or). Cependant, la réalisation de ses activités qui devraient être en conformité avec les règlementations en vigueur en matière d'environnement, d'urbanisme, d'installations classées et de protection du patrimoine forestier comme stipulée dans le code minier (article 143), est peu en adéquation avec cette législation. Cet état de fait favorise souvent des problèmes environnementaux et sanitaires (SGS, 2016, p. 18). Cette étude a pour objectif global d'analyser l'incidence des déchets toxiques produits dans la mine d'or de Tongon sur la santé des travailleurs. Plus spécifiquement, il s'agit d'une part, d'identifier les différents déchets produits dans la mine d'or de Tongon et leur contenu, et d'autre part, de déterminer les pathologies récurrentes diagnostiquées chez les travailleurs de la mine en relation avec ces déchets. Dans cette étude, seuls les déchets miniers (ou industriels) ont été retenus car ils sont dangereux pour la santé humaine et constituent une menace pour l'environnement (Kone, 2016, p. 63).

\section{Outils et méthodes}

\subsection{Présentation de la zone d'étude}

La mine d'or de Tongon est située au nord de la Côte d'Ivoire, dans la région du Poro. Le site actuel de la société Tongon SA s'étend sur environ 2000 ha dont plus de 50\% est occupé par le couvert végétal. Elle compte total au 1307employés (Kone, 2016, p. 42).

Cette mine est exploitée par la société Sud-Africaine Randgold Resources Ltd qui détient $89 \%$ du capital contre $10 \%$ pour l'Etat de Côte d'Ivoire et $1 \%$ pour une entreprise locale (SGS, 2016, p.31). Randgold Resources Ltd dispose de 5 permis dont 4 permis de recherche et 1 d'exploitation couvrant un total de $6000 \mathrm{~km}^{2}\left(\mathrm{MEMPD}^{10}, 2015\right.$, p.94) (figure $1)$.

${ }^{10}$ Ministère d'Etat, Ministère du Plan et du Développement 


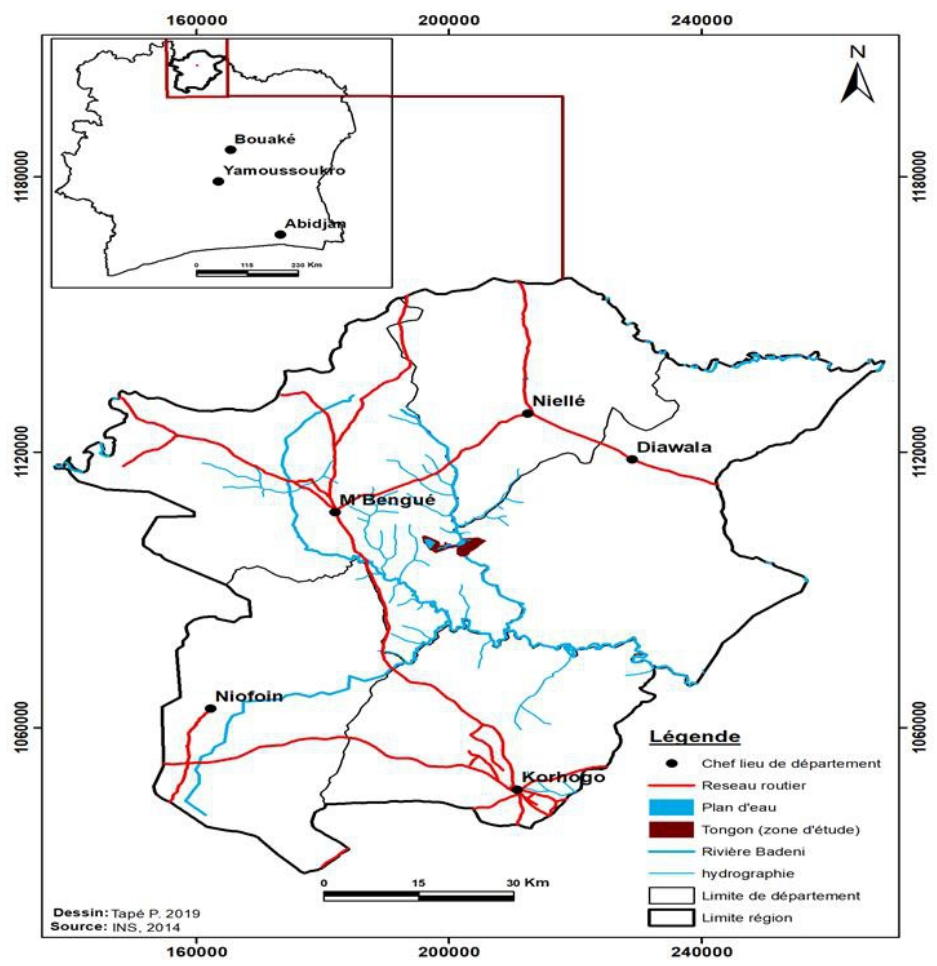

Figure 1: Localisation géographique de la mine d'or de Tongon

\subsection{Technique et collecte de données}

La méthodologie utilisée pour collecter les données s'est appuyée sur la revue documentaire, la réalisation des interviews semi-structurées et l'administration des questionnaires. La revue bibliographique a consisté en la consultation des ouvrages divers (mémoires, thèses, rapports, etc.) sur internet, dans les bibliothèques de l'Université Peleforo Gon Coulibaly et de l'Institut de Géographie Tropicale (IGT). Cette revue documentaire s'est également étendue à la Société des Mines (SODEMI), à l'Agence Nationale de l'Environnement (ANDE), au Ministère des Mines et de l'Energie, aux structures d'audit, de contrôle et de certification environnementale dans le domaine minier (Bureau Veritas, Société Générale de Surveillance (SGS)) ainsi qu'aux services de santé de la mine de Tongon et au Centre Hospitalier Régional (CHR) de Korhogo. La capitalisation de toutes ces informations a permis de comprendre les concepts de déchets en général et de déchets miniers en particulier, le fonctionnement d'une mine d'or, les techniques utilisées, les différents matériels et équipements utilisés et la règlementation en matière d'exploitation minière et aurifère.

Les entretiens semi-directifs ont été réalisés auprès des services de la direction des ressources humaines, de l'environnement et de ceux de la santé. Ces entretiens ont porté sur l'effectif des travailleurs, leur répartition par 
direction, les types de déchets produits, les produits chimiques utilisés, les dispositions prises aux plans environnemental et sanitaire, les maladies diagnostiquées chez les employés de la mine et leur prise en charge.

Quant à l'enquête, des questionnaires ont été administrés aux travailleurs de la mine sur la base d'un sondage stratifié. L'échantillon d'étude est de 297 travailleurs. La taille de l'échantillon est déterminée à partir de la formule suivante :

$$
\mathrm{n}=\frac{\mathrm{Z}^{2}(\mathrm{PQ}) \mathrm{N}}{\mathrm{e}^{2}(\mathrm{~N}-1)+\mathrm{Z}^{2}(\mathrm{PQ})}
$$

Avec: $n=$ taille d'échantillon requise, $\mathrm{Z}=$ niveau de confiance à $95 \%$ (coefficient de marge 1,96), $\mathrm{p}=$ prévalence des pathologies liées à l'exploitation minière, $\mathrm{e}=$ marge d'erreur à $5 \%$ et $\mathrm{Q}=1-\mathrm{P}$.

Les différentes strates utilisées sont la Direction Générale, la Direction des Opérations et le service Supports Généraux. Les travailleurs enquêtés sont répartis sur la base du choix d'une répartition proportionnelle comme suit : Direction Générale (3 enquêtés), Direction des Opérations (208 enquêtés) et le service Supports Généraux (86 enquêtés). Ces 297 unités statistiques représentent $23 \%$ de l'effectif total des employés.

Toutes les données collectées ont été saisies à l'aide du tableur Excel de logiciel Microsoft office 2010.

\section{Résultats}

Ces résultats concernent les différents déchets miniers (ou industriels) produits par la mine de Tongon, leur contenu ainsi que les différentes pathologies et signes cliniques liées aux répercussions de ces déchets.

\subsection{Typologie des déchets industriels produits dans la mine de Tongon}

Les déchets miniers sont définis comme des déchets dangereux inertes (ou banals) résultant d'une activité de production industrielle, artisanale, commerciale ou tertiaire dont l'élimination doit incomber financièrement aux producteurs (Kone, 2016, p. 63). Ces déchets contiennent des substances chimiques telles que l'amiante, l'arsenic et/ou le plomb constitués de boues de peinture, d'hydrocarbure, des produits de vidange provenant des ateliers de traitement de surface. Dans la mine de Tongon, l'on retrouve trois grands types de déchets industriels (ou miniers) : les déchets de roches stériles (résidus miniers), la boue et la terre contaminée.

\subsubsection{Déchets de roches stériles}

Les déchets de roches stériles sont des rejets d'origine géologique produits par les opérations minières. Ces résidus miniers proviennent de la carrière de l'or. Ils ne contiennent pas de minerais d'or mais des minéraux associés tels que le sulfure de fer et l'arsenic. Ils sont en général entreposés 
dans des empilements appelés haldes à stérile. La figure 2 ci-dessous montre une vue de stockage des roches stériles.

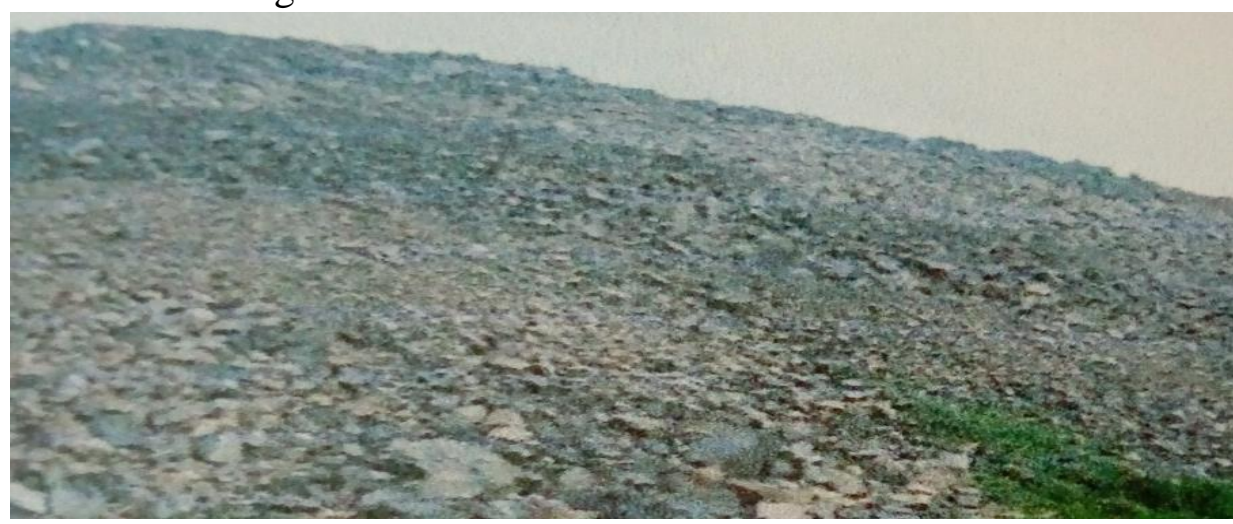

Figure 2 : Vue de roches stériles (Source : Enquête de terrain de Tape \& Coulibaly, 2019)

Ces résidus miniers proviennent des puits d'or. Ils sont composés de minéraux naturels très souvent nuisibles à la santé et à l'environnement.

La figure 3 ci-dessous montre les différentes étapes de l'évolution de la quantité de roches stériles produites.

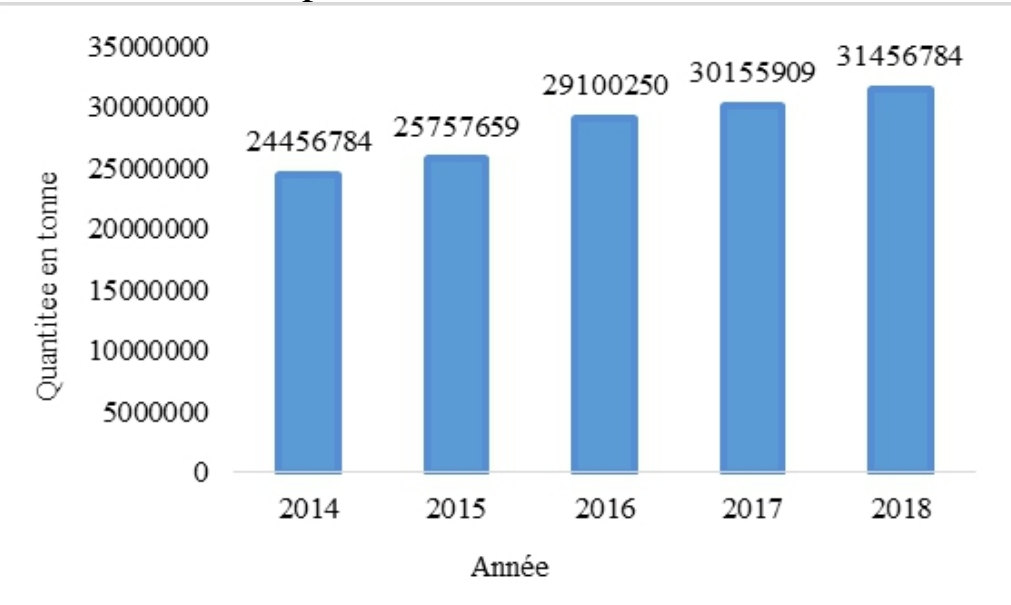

Figure 3: Evolution de la production annuelle des roches stériles de 2014 à 2018

(Source : Enquête de terrain de Tape \& Coulibaly, 2019)

La production de ces déchets est passée de 24456784 tonnes en 2014 à 31456784 tonnes en 2018, soit une augmentation de 28,62\% selon le département mining. Cette augmentation est due à l'accroissement de la production et à la profondeur des fouilles.

\subsubsection{Boue issue des minerais}

La boue (talling solution) provient du traitement du minerai (la pulpe). Elle est issue des phases de traitement du minerai. Cette boue est rejetée dans 
le parc à boue situé à $7 \mathrm{~km}$ de l'usine. La figure 4 ci-dessous montre le stockage des boues à la mine d'or de Tongon.

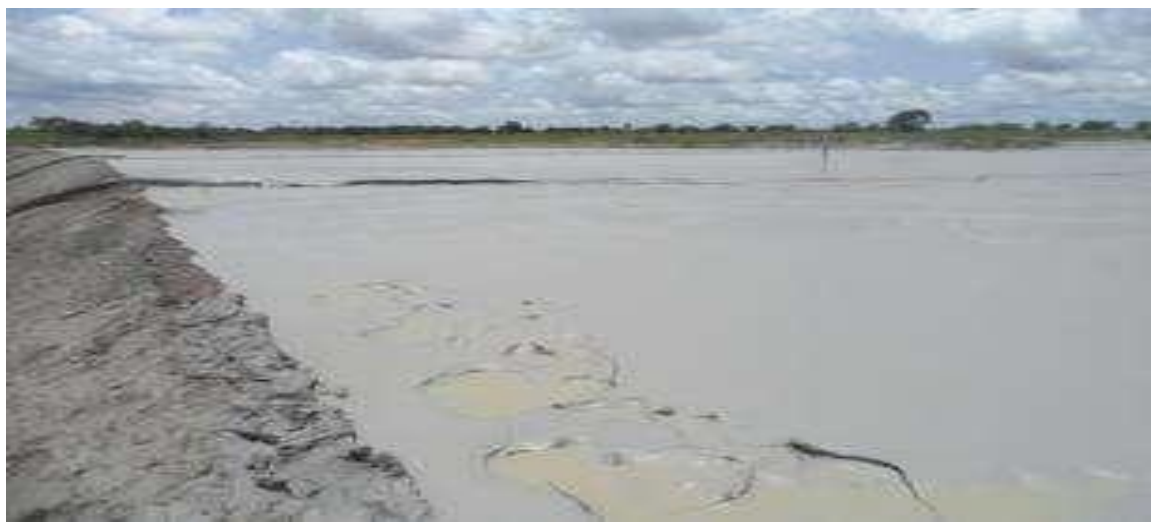

Figure 4: Vue de stockage des boues au niveau du parc à boue de Tongon

(Source : SGS, 2014, p.71)

La figure 5 indique l'évolution de la quantité de boue rejetée lors du traitement du minerai. Cette quantité de boue rejetée a connu une relative croissance. De $4190597 \mathrm{~m}^{3}$ en 2014, elle est passée à $4689522 \mathrm{~m}^{3}$ en 2015 et a atteint $5188447 \mathrm{~m}^{3}$ en 2016 puis 7465785 en 2018 .

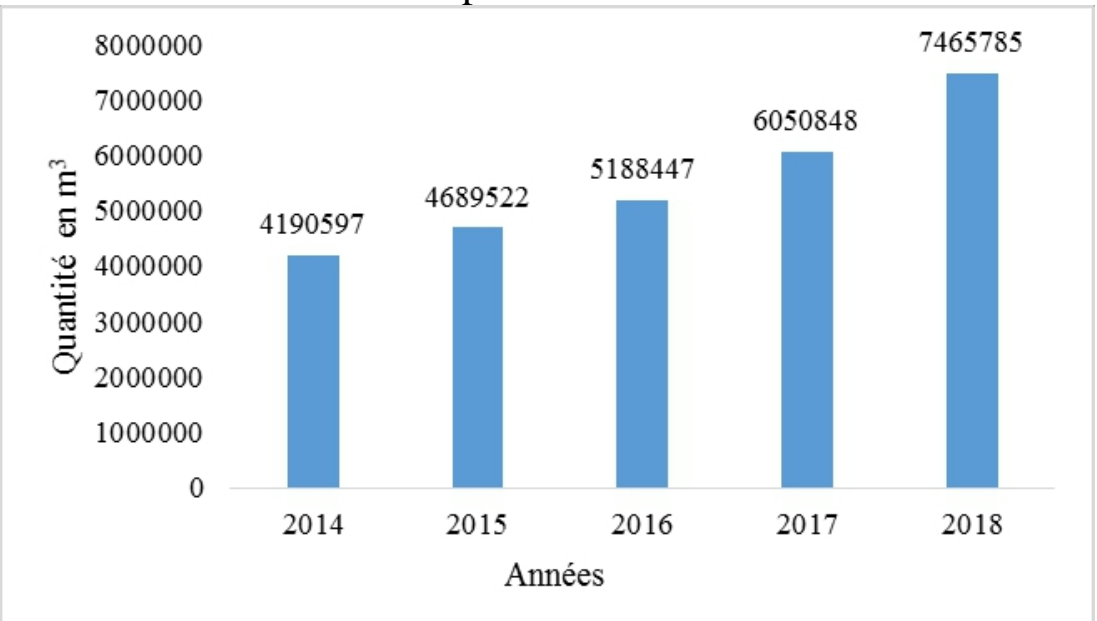

Figure 5: Evolution de la quantité de boue rejetée dans le parc à boue de 2014 à 2018 (Source : Enquête de terrain de Tape \& Coulibaly, 2019)

\subsubsection{Terre contaminée}

La terre contaminée à Tongon est un type de déchets issus des déversements de produits chimiques et d'hydrocarbures. Elle constitue un danger pour la santé humaine et environnementale. La figure 6 ci-dessous montre l'évolution de la quantité des terres contaminées entre 2014 et 2018. Cette augmentation des terres contaminées s'explique par la densification des 
travaux sur les sites d'exploitation et la dégradation des engins. En effet, de 1234,78 tonnes en 2014, la quantité de terre contaminée est passée à 1656,03 tonnes en 2015 et a atteint 1952,70 tonnes en 2016. Avec l'évolution des travaux la quantité de terres contaminées a connu une importante augmentation en passant de 2149,56 tonnes en 2017 à 2446,23 tonnes en 2018, soit une évolution de $13,80 \%$.

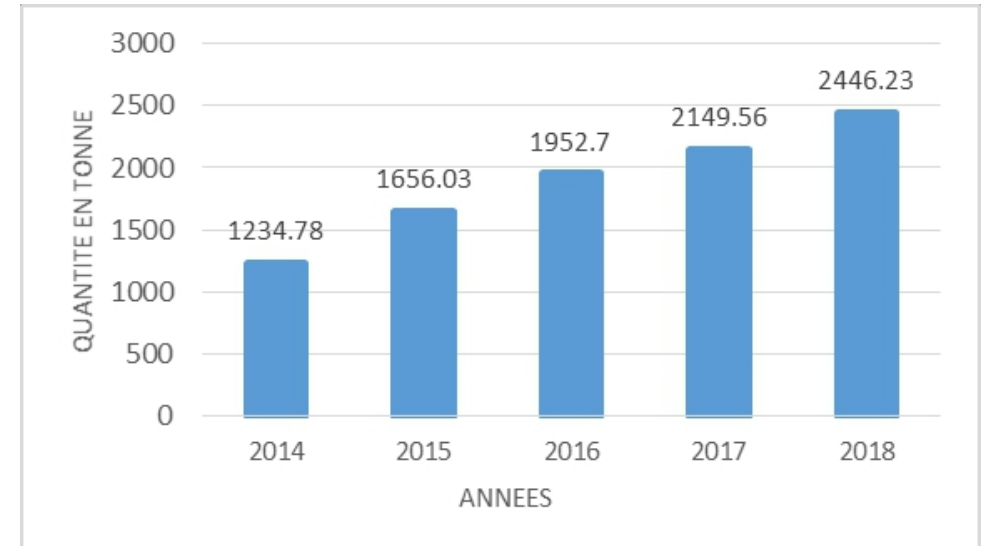

Figure 6: Evolution de la quantité de terre contaminée de 2014 à 2018

(Source : Enquête de terrain de Tape \& Coulibaly, 2019)

\subsection{Contenu des déchets miniers produits dans la mine de Tongon et les incidences sur la santé}

Les déchets miniers à Tongon sont nombreux et dangereux. Cette dangerosité est imputable à l'utilisation massive des intrants chimiques dangereux pour la santé dans l'exploitation aurifère. L'étude montre que, des produits chimiques tels que le cyanure de sodium $(\mathrm{NaCN})$, l'acide chlorhydrique $(\mathrm{HCI})$ et le peroxyde d'hydrogène $\left(\mathrm{H}_{2} \mathrm{O}_{2}\right)$ y sont utilisés. Le cyanure de sodium sert à dissoudre l'or dans du gisement, alors que l'acide chlorhydrique est utilisé pour prélever le calcium du carbone. Enfin, le peroxyde d'hydrogène est utile pour l'oxydation. Dans la liste des produits chimique utilisés, il y a également la chaux pour le contrôle de PH (CIL) et le nitrate d'ammonium pour le mélange des explosifs. Une fois ces produits utilisés, les résidus se retrouvent inéluctablement dans les différents déchets produits. Ainsi, les roches stériles qui proviennent de la carrière de l'or contiennent des minéraux associés tels que le sulfure de fer et l'arsenic. Quant à la boue, elle renferme des additifs chimiques, minéraux ou organiques que sont le cyanure $(\mathrm{CN}-)$, le plomb $(\mathrm{Pb})$, le fer $(\mathrm{Fe})$ et le nitrate $(\mathrm{Na})$ hautement nocives pour la santé. Exposés à ces produits chimiques sur un site minier, les travailleurs développent des pathologies et signes cliniques tels que des étourdissements, la nausée et des vomissements, des maux de tête, des convulsions, des hallucinations, des respirations rapides et profondes, des irritations de la peau, des pressions sanguines basses, des dommages au 
cerveau, l'encéphalopathie, neuropathies périphériques, cancer, des atteintes neurologiques et rénales. Ses effets hématologiques se résument en des troubles de la synthèse de l'hème, l'anémie et aux hématies à granulations basophiles (Lavigne et al., 2004, pp.9-10; Bismuth et al., 2000, pp.75-150).

\subsection{Situation sanitaire dans la mine d'or de Tongon}

La situation sanitaire dans la mine d'or de Tongon est appréciée à travers la fréquence de visite de sa structure de soins par les travailleurs, les pathologies diagnostiquées, les symptômes de certaines pathologies (signes cliniques suspectés) en relation avec les sites de travail des enquêtés.

\subsubsection{Fréquentation de la clinique de la mine d'or}

Les données relatives à la dynamique annuelle de la fréquentation de la clinique de Tongon sont consignées dans le tableau 1 ci-dessous.

Tableau 1: Bilan de fréquentation de la clinique de la mine d'or de Tongon

\begin{tabular}{|c|c|c|}
\hline Années & Nombre de consultations & $\begin{array}{c}\text { Taux de } \\
\text { variation }\end{array}$ \\
\hline 2016 & 1009 & $-15 \%$ \\
\cline { 2 - 2 } 2017 & 857 & $+40 \%$ \\
\hline 2018 & 1200 & +2019 \\
\hline
\end{tabular}

Source : Enquête de terrain de Tape \& Coulibaly, 2019

Le tableau 1 indique que, la fréquentation des services de santé de Tongon a connu une baisse de $15 \%$ entre 2016 et 2017. En revanche, cette fréquentation a connu une augmentation de $40 \%$ entre 2017 et 2018. Selon le service de santé au sein de Tongon, les pathologies les plus fréquemment rencontrées chez les salariés sont les gastro-entérites (15\%), la carie et affections bucco-dentaire (1\%), le paludisme (16\%), les maladies respiratoires $(11 \%)$, les céphalées $(23 \%)$, les infections sexuelles $(0,5 \%)$, les otites $(5 \%)$, les traumatismes $(1 \%)$, les plaies et affections de la peau (11\%), les affections de l'œil (9\%), les maladies cardiovasculaires $(0,5 \%)$ et les fièvres d'origine indéterminée $(7 \%)$.

\subsubsection{Pathologies et signes cliniques identifiés chez les enquêtés}

Les pathologies et les signes cliniques identifiées au cours de l'étude chez les individus enquêtés sont reportés dans le tableau 2. Ce sont des maladies dont souffraient les enquêtés au moment de l'étude ou dont ils ont souffert au cours des 12 derniers mois précédent l'enquête. A défaut de prélèvements pour des analyses biomédicales, certains symptômes de pathologies ont été classés sous le vocable d'irritations avec l'aide des spécialistes en santé et maladies professionnelles. 
Tableau 2: Pathologies et signes cliniques identifiés chez les individus enquêtés

\begin{tabular}{|l|c|c|}
\hline Pathologies ou signe symptomatiques & $\begin{array}{c}\text { Effectif d'enquêtés } \\
\text { développant une pathologie }\end{array}$ & Proportion \\
\hline $\begin{array}{l}\text { Irritations oculaires } \\
\text { (conjonctivite, larmoiement, douleurs } \\
\text { oculaires, baisse de la vision oculaire) }\end{array}$ & 6 & $8 \%$ \\
\hline $\begin{array}{l}\text { Irritations respiratoires } \\
\text { (toux, dyspnée, odème pulmonaire, } \\
\text { saignements de nez, ulcérations nasales, } \\
\text { bronchites chroniques, détresse respiratoire) }\end{array}$ & 8 & $11 \%$ \\
\hline $\begin{array}{l}\text { Irritation digestive } \\
\text { (douleurs buccales, des vomissements } \\
\text { fréquents parfois sanglants, détresse } \\
\text { respiratoire, odème laryngé) }\end{array}$ & 10 & $14 \%$ \\
\hline $\begin{array}{l}\text { Irritations auditives } \\
\text { (otites) }\end{array}$ & 3 & $4 \%$ \\
\hline Céphalées (fréquentes) & 18 & $24 \%$ \\
\hline Lésions caustiques & 13 & $17 \%$ \\
\hline Paludisme & 16 & $100 \%$ \\
\hline Total & 74 & \\
\hline
\end{tabular}

Source: Enquête de terrain de Tape \& Coulibaly, 2019

Les résultats des interviews semi-directifs montrent que 74 des 297 individus interrogés développent une pathologie ou un signe clinique d'une pathologie. Cette population de salariés malades représente $25 \%$ des enquêtés. Parmi les salariés enquêtés atteints d'une pathologie (tableau 2), 24\% souffrent fréquemment de céphalées et $22 \%$ du paludisme sous toutes ses formes. Les lésions caustiques affectent $17 \%$ d'entre eux contre $14 \%$ et $11 \%$ respectivement pour des cas d'irritations digestives et respiratoires. Les cas d'irritations oculaires ne concernent que $8 \%$ de salariés enquêtés.

\subsubsection{Répartition des individus développant une pathologie selon la zone d'intervention sur le lieu de travail}

Les données concernant les services enquêtés atteints d'une pathologie ou qui portent des signes cliniques d'une pathologie sont présentées dans le tableau 3 ci-dessous.

Tableau 3: Nombre de salariés enquêtés ayant une pathologie selon les services

\begin{tabular}{|c|c|c|c|c|}
\hline Directions & Services (départements) & Effectif & \multicolumn{2}{|c|}{ Proportion } \\
\hline \multirow{4}{*}{$\begin{array}{c}\text { Direction } \\
\text { des } \\
\text { Opérations }\end{array}$} & Opération minière & 14 & $19 \%$ & \multirow{4}{*}{$74 \%$} \\
\hline & Ressources minérales & 19 & $26 \%$ & \\
\hline & Usine de production & 13 & $17 \%$ & \\
\hline & Maintenance & 9 & $12 \%$ & \\
\hline \multirow{3}{*}{$\begin{array}{l}\text { Supports } \\
\text { Généraux }\end{array}$} & $\begin{array}{l}\text { Hygiène Sécurité } \\
\text { Environnement (HSE) }\end{array}$ & 7 & $9 \%$ & \multirow{3}{*}{$26 \%$} \\
\hline & Sécurité & 5 & $7 \%$ & \\
\hline & Ressources humaines & 2 & $3 \%$ & \\
\hline
\end{tabular}




\begin{tabular}{|c|l|c|c|c|}
\hline & Logistique & 3 & $4 \%$ & \\
\cline { 2 - 4 } & Communauté & 2 & $3 \%$ & \\
\hline \multicolumn{2}{|c|}{ Total } & 74 & $100 \%$ & $100 \%$ \\
\hline
\end{tabular}

Les résultats obtenus des questionnaires révèlent que $74 \%$ des individus enquêtés en situation morbide travaillent à la Direction des Opérations de la mine. Ils souffrent des irritations respiratoires (toux, saignements de nez, ulcérations nasales, bronchites chroniques, détresse respiratoire), oculaires (douleurs oculaires, baisse de la vision oculaire), digestives (douleurs buccales, des vomissements fréquents parfois sanglants, détresse respiratoire, œdème laryngé), auditives (otites fréquentes) et des céphalées fréquentes. C'est à cette direction qu'appartiennent les départements les plus importants de Tongon. Ces sont des départements qui s'occupent de l'exploitation, du contrôle des teneurs, de la manipulation des intrants chimiques, de la production et de l'entretien de tous les appareils et outils sur les sites de Tongon. Les agents de cette direction seraient donc à priori plus exposés aux risques de pathologies que leurs collègues des autres directions qui s'occupent dans la plupart des cas des tâches administratives.

\section{Discussion}

A la mine de Tongon, les déchets qui proviennent des activités sont multiples et contiennent des produits chimiques très nocifs pour l'homme. Ces déchets qualifiés de dangereux ont également été identifiés par Kone (2016) qui a travaillé sur la gestion des déchets de cette exploitation minière. Cette étude a révélé qu'en plus des roches stériles, de la boue et des terres contaminées, il y a aussi les déchets médicaux qui pourraient être classés comme déchets dangereux au niveau de la mine de Tongon. De plus, selon cette même étude, l'augmentation de la quantité des terres contaminées est due à la vétusté des engins de l'usine, au rejet de gaz et au déversement du gasoil entrainant ainsi la pollution de l'air, des sols, des eaux de surfaces et souterraines (Kone, 2016, pp.63-67). Les effets des intrants chimiques que contiennent ces déchets sur la mine de Tongon ont également été identifiés par Digby et al.(2008, p. 32).

L'analyse du rapport d'audit environnemental et réglementaire au niveau de la mine d'or révèle qu'en dépit des conditions de travail relativement bonnes (SGS, 2016, pp.145-48), les travailleurs sont exposés aux risques d'intoxication et aux maladies liés à l'utilisation massives des intrants chimiques dans l'extraction aurifère et à la gestion des déchets générés. Les lésions caustiques (brûlantes) et les irritations digestives, respiratoires, oculaires et auditives dont certains agents sont victimes, ont été rapportées par Digby et al. (2008, pp.252-276) et SGS (2016, pp.61-92) ainsi que les mécanismes d'atténuation de diffusion de leur impact. En outre, il semble 
avoir une similarité entre les pathologies dont souffrent les travailleurs de la mine d'or Tongon et ceux de la zone aurifère de Kéniéba au Mali (Seydou, 2001 in Ouedraogo, 2012, p.24). En effet, sur ce site aurifère, on utilise les mêmes intrants chimiques et dangereux qu'à Tongon (cyanure de sodium, peroxyde d'hydrogène, nitrate de plomb, chaux, nitrate d'ammonium, acide chlorhydrique). Un rapport portant sur «Les effets du plomb sur la santé » du centre canadien d'hygiène et de sécurité au travail révèle également que des symptômes comprenant des maux de tête, de la fatigue extrême, des nausées et des crampes abdominales, surviendraient après une exposition modérée au plomb inorganique après douze heures de travaux de décapage. A cela, il faut ajouter des douleurs articulaires, de l'anorexie (absence, perte d'appétit), une inflammation de la muqueuse de l'estomac (gastrite) et une diminution de la miction CCHST, 2019, pp.2-4). Il en est de même pour le cyanure d'hydrogène. Une étude menée au Québec révèle que, l'absorption du cyanure d'hydrogène par les poumons, la peau et par ingestion de façon accidentelle en situation de travail, entraine des symptômes immédiats ou différés comme les fortes céphalées, des vertiges, des étourdissements et des nausées (Lavigne et al., 2004, pp.5-9). Toutefois, selon Host et al. (2006, p.1), l'évaluation des risques sanitaires associés à une exposition environnementale est complexe et faite d'incertitudes. Dans la plupart des cas, il est difficile de les quantifier précisément et de prendre en compte d'éventuelles interactions. Ces expositions, susceptibles de provoquer des maladies, n'induisent pas des pathologies qui leur sont spécifiques, dans la mesure où d'autres facteurs liés aux comportements des individus (tabac, alcool) ou aux antécédents génétiques peuvent en être la cause. Par ailleurs, ces maladies se manifestent chez les individus généralement longtemps après leur contact avec le (ou les) agent(s) dangereux. Il n'est donc pas aisé de relier avec certitude un facteur environnemental et un effet sanitaire qui du reste, est généralement multifactorielle.

\section{Conclusion}

Considérée comme la plus grande exploitation aurifère de Côte d'Ivoire, la mine d'or de Tongon produit des déchets miniers ou industriels caractérisés de dangereux car contenant des intrants chimiques très nocifs pour la santé humaine et environnementale. Constitués de roches stériles (résidus miniers), de la boue et de terre contaminée, ces déchets miniers contiennent du cyanure, de l'acide chlorhydrique, de l'arsenic, du plomb, du fer, du nitrate, du peroxyde d'hydrogènes. Les travailleurs de cette mine sont régulièrement exposés à certaines de ces substances nocives. Cela constitue des facteurs de risques pour la santé de ceux-ci. Les pathologies et signes cliniques rencontrés chez les travailleurs mettent en exergue l'impact négatif des conditions de travail et des rejets miniers sur la santé des employés de la mine d'or. Ainsi, 
pour réduire les effets néfastes des activités de la mine de Tongon sur la santé des individus, le renforcement des mesures de protection et de prévention s'avèrent nécessaires.

\section{References:}

1. Bamba N. L. \& Lauzon N. M. (2008). Rapport Afrique de l'Ouest, Nigeria Abuja, CEDEAO, 98p.

2. Bismuth C., Baud F., Conso F., Dally S., Frejaville J. P., Garnier R., Jaeger A. (2000). Toxicologie clinique. Paris, Médecine-Sciences, Flammarion, $5^{\text {ème }}$ édition, 1092 p.

3. Chambre de commerce et d'industrie (2017). Fiche sectorielle : les mines en bref. Abidjan, Chambre de Commerce et d'Industries de Côte d'Ivoire [en ligne] Consultée le 25/08/2019 sur http: //www.cci.ci

4. Centre canadien hygiène et de sécurité au travail (2019). Fiche d'information réponses SST. Canada, Centre Canadien d'Hygiène et de Sécurité au Travail, [en ligne] Consultées le 25/08/2019 sur http/www.cchst.ca/ ashanswers/about.html

5. Dicko A. A. (2011). Réalisation d'un audit environnemental de la mine d'or de Morila au Mali : cas de cessation d'activité. Mémoire de Master en Ingénierie de l'Eau et de l'Environnement option Environnement. Institut International d'Ingénierie de l'Eau et de l'Environnement, Ouagadougou Burkina Faso, 57 p.

6. Digby W. \& Associates (2008). Evaluation des impacts environnementaux (EIE) et le Plan de Gestion Environnementale (PGE) pour le projet de la mine d'or de Tongon. Afrique du Sud, Randburg 2125, Environmental Solutions Provides, 286 p.

7. Host S., Camard J-P., Camard A. F., Lefranc A., Grémy I. (2006). L'évaluation des risques sanitaires : principe et méthode. France, Observation Régional de Santé d'ile de France (ORS), ISBN, 2-73711564-7, pp1-6

8. Kone B. (2018). Communiqué du Conseil des Ministres du 24 Janvier 2018. Abidjan, [en ligne] Consultées le 25/08/2019 sur http/www.presidence.ci/communique-du-conseil-des-ministres-dumercredi-24janvier-2018/

9. Kone K. (2016). La gestion des déchets de l'exploitation minière de Tongon dans la Sous-préfecture de M'Bengué. Côte d'Ivoire, Bouaké, Université Alassane Ouattara de Bouaké, Département de Géographie, Mémoire Master de Géographie, 139 p.

10. Lavigne J., Roy L-A., Lefebvre L. (2004). Guide toxicologique pour les urgences en santé environnementale. Les cyanures: Cyanure d'hydrogène. Section B-1. Québec, Montréal, Direction de la Santé Publique de Montréal, 25 p. 
11. Ministère d'état, Ministère du Plan et du Développement (2015). Etudes monographiques et économiques des districts de Côte d'Ivoire : District des Savanes. Côte d'Ivoire, Korhogo, Direction Régionale du Ministère du Plan et du Développement, 306 p.

12. Ouedraogo I. (2012). Impact de l'exploitation minière industrielle sur la santé humaine et environnementale au Burkina Faso: cas de la mine d'or de Essakane SA. France, Université de Versailles Saint Quentin en Yvelines, Master Pro 2 en Sciences de la Santé, de l'Environnement, du Territoire et de la Société (SSEnTS), $44 \mathrm{p}$.

13. Sekongo P. K. (2015). Etude de la fiabilité des concasseurs primaires de l'usine de traitement de Tongon. Abidjan, Groupe CSI Pôle Polyethnique, Mémoire de fin de cycle Master II, $73 \mathrm{p}$.

14. Société Générale de Surveillance (2016). Rapport d'audit environnemental réglementaire de la mine d'or de Tongon. Abidjan, SGS Côte d'Ivoire, $350 \mathrm{p}$.

15. Union international pour la conservation de la nature/Programme des Aires protégées d'Afrique centrale et de l'ouest (2011). Evolution du secteur minier en Afrique de l'Ouest. Quel impact sur le secteur de la conservation? Ouagadougou, Burkina Faso, UICN/PACO, numéro $10,177 \mathrm{p}$. 